STRUCTURAL BIOLOGY COMMUNICATIONS

ISSN 2053-230X

Received 8 February 2018

Accepted 19 March 2018

Edited by K. K. Kim, Sungkyunkwan University School of Medicine, Republic of Korea

Keywords: crystal structure; glyceraldehyde-3phosphate dehydrogenase; GAPDH;

cryoprotectants; trehalose.

PDB reference: glyceraldehyde-3-phosphate dehydrogenase, $5 z a 0$

Supporting information: this article has supporting information at journals.iucr.org/f

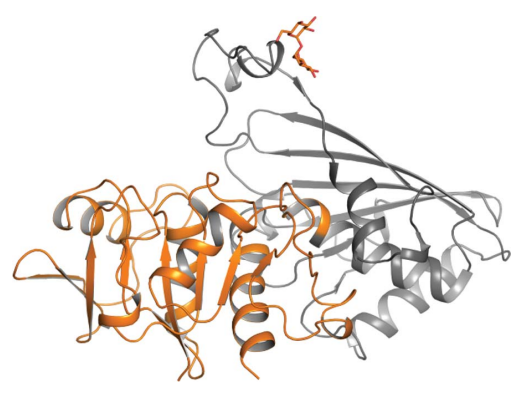

OPEN $\odot$ ACCESS

\section{A cryoprotectant induces conformational change in glyceraldehyde-3-phosphate dehydrogenase}

\author{
Yong Ju Kim*
}

\begin{abstract}
Department of Herbal Medicine Resources, College of Environmental and Bioresource Sciences, Chonbuk National University, Iksan 54596, Republic of Korea, and Department of Lifestyle, College of Environmental and Bioresource Sciences, Chonbuk National University, Iksan 54596, Republic of Korea. *Correspondence e-mail:

nationface@jbnu.ac.kr
\end{abstract}

Glyceraldehyde-3-phosphate dehydrogenase (GAPDH), a glycolytic enzyme, catalyses the conversion of D-glyceraldehyde 3-phosphate to 1,3-bisphosphoglycerate. While mammalian and yeast GAPDHs are multifunctional proteins that have additional functions beyond those involved in glycolysis, including reactions related to nuclear RNA transport, DNA replication/repair, membrane fusion and cellular apoptosis, Escherichia coli GAPDH (ecGAPDH) has only been reported to function in glycolysis. The S-loop of GAPDH is required for interaction with its cofactor and with other proteins. In this study, the threedimensional crystal structure of GAPDH treated with trehalose is reported at $2.0 \AA$ resolution. Trehalose was used as a cryoprotectant for the GAPDH crystals. The structure of trehalose-bound $e c \mathrm{GAPDH}$ was compared with the structures of both $\mathrm{NAD}^{+}$-free and $\mathrm{NAD}^{+}$-bound $e c \mathrm{GAPDH}$. At the S-loop, the bound trehalose in the GAPDH structure induces a $2.4^{\circ}$ rotation compared with the $\mathrm{NAD}^{+}$-free $e c \mathrm{GAPDH}$ structure and a $3.1^{\circ}$ rotation compared with the $\mathrm{NAD}^{+}$-bound $e c \mathrm{GAPDH}$ structure.

\section{Introduction}

Many crystals of biological macromolecules are sensitive to $\mathrm{X}$-rays near room temperature and frequently suffer from radiation damage, especially when $\mathrm{X}$-ray experiments are carried out on highly intense synchrotron beamlines (Hope, 1990). To prevent radiation damage from X-rays (Watenpaugh, 1991; Rodgers, 1994; Low et al., 1966) and to facilitate the transport and simple storage of protein crystals, many cryoprotectants have been developed (Garman, 1999, 2003) and many cryoprotectant products are commercially available, such as CryoPro from Hampton Research.

In general, cryoprotectants are small polyols and organics, such as glycerol, ethylene glycol, 1,2-propanediol, diethylene glycol, 2-methyl-2,4-pentanediol, dimethyl sulfoxide or other nonvolatile alcohols (Pflugrath, 2015). Low-molecular-weight polyethylene glycols (PEGs), such as PEG 200 or PEG 400, are suitable cryoprotectants in many cases. Higher molecularweight PEGs can also be used, albeit with more difficulty than lower molecular-weight PEGs. High concentrations of salts, such as lithium formate (Rubinson et al., 2000), or carboxylic acids, such as malonate, can also be used (Holyoak et al., 2003), as can sugars such as sucrose, trehalose, sorbitol, xylitol or glucose (Pflugrath, 2015).

Glyceraldehyde-3-phosphate dehydrogenase (GAPDH) is an enzyme that catalyses the conversion of glyceraldehyde 3-phosphate to 1,3-bisphosphoglycerate using $\mathrm{NAD}^{+}$as a cofactor (Baker et al., 2014) and is known to be a moonlighting 
Table 1

Crystallization conditions.

\begin{tabular}{ll}
\hline Method & Hanging-drop vapour diffusion \\
Plate type & 24-well plates \\
Temperature $\left({ }^{\circ} \mathrm{C}\right)$ & 4 \\
Protein concentration $\left(\mathrm{mg} \mathrm{ml}^{-1}\right)$ & 20 \\
Buffer composition of protein solution & $20 \mathrm{~m} M$ Tris pH 7.5, 0.1 M NaCl \\
Composition of reservoir solution & $0.1 \mathrm{M}$ MES pH 5.5-6.5, \\
& $2.8 \mathrm{M}$ ammonium sulfate \\
Volume and ratio of drop & $2 \mu \mathrm{l}: 2 \mu \mathrm{l}$ \\
Volume of reservoir $(\mathrm{ml})$ & 1
\end{tabular}

protein (Savreux-Lenglet et al., 2015). This enzyme plays multiple roles in the regulation of mRNA stability (Zhou et al., 2008), intracellular membrane trafficking (Sirover, 2012), iron uptake and transport (Zaid et al., 2009), DNA replication and repair (Zheng et al., 2003), and nuclear RNA transport (Dastoor \& Dreyer, 2001). In particular, yeast GAPDH is inhibited by trehalose (Araiza-Olivera et al., 2010). An important region of GAPDH is the S-loop (residues 178-201), a long, winding region of the enzyme that is known to interact with some proteins (Kosova et al., 2017; Duée et al., 1996). The S-loop region of $\mathrm{NAD}^{+}$-free GAPDH has a very flexible shape and thus does not show clear electron density (Ferreira-daSilva et al., 2006; Querol-García et al., 2017). On the other hand, in NAD ${ }^{+}$-bound GAPDH S-loop fixation occurs by the formation of a complex with the coenzyme $\mathrm{NAD}^{+}$(Kitatani et al., 2006).

GAPDHs have been isolated from bacteria, archaea, prokaryotes, eukaryotes, plants and mammals. Under normal cellular conditions, GAPDH primarily has a tetrameric conformation composed of four identical $35 \mathrm{kDa}$ subunits (Nicholls et al., 2012; Frayne et al., 2009). GAPDH consists of an $\mathrm{NAD}^{+}$-binding domain (residues 2-148 and 312-330), which is composed of $\alpha / \beta$ dinucleotide-binding folds or Rossmann folds, and a catalytic domain (residues 149-311), which is composed of eight antiparallel $\beta$-sheets with an $\alpha$-helix and several short loops (Duée et al., 1996; Yun et al., 2000). The activity of GAPDH requires the binding of $\mathrm{NAD}^{+}$.

In this study, the purification, crystallization and cryoprotection of Escherichia coli GAPDH (ecGAPDH) and the three-dimensional structure of trehalose-bound ecGAPDH are described. The GAPDH crystals were cryoprotected with a solution containing $15 \%$ of the disaccharide trehalose.

\section{Materials and methods}

\subsection{Preparation of GAPDH}

E. coli BL21 (DE3) cells were grown on LB agar medium containing $100 \mu M$ ampicillin. A single colony was cultivated in $100 \mathrm{ml} \mathrm{LB}$ medium overnight at $37^{\circ} \mathrm{C}$. The next day, a main culture was cultivated in $11 \mathrm{LB}$ medium for $4 \mathrm{~h}$ at $37^{\circ} \mathrm{C}$. The cultured cells were harvested by centrifugation $\left(4^{\circ} \mathrm{C}\right.$, $\left.8000 \mathrm{rev} \mathrm{min}^{-1}, 5 \mathrm{~min}\right)$. The harvested cells were resuspended in buffer $A$ (20 m $M$ Tris, $130 \mathrm{~m} M \mathrm{NaCl} \mathrm{pH} \mathrm{7.5).} \mathrm{The} \mathrm{cells} \mathrm{were}$ disrupted by sonication on ice and the cell lysate was then separated from the cell debris by centrifugation $\left(4^{\circ} \mathrm{C}\right.$, $20000 \mathrm{rev} \mathrm{min}^{-1}, 20 \mathrm{~min}$ ). The supernatant was loaded onto a
Table 2

Data collection and processing.

Values in parentheses are for the outer shell.

\begin{tabular}{ll}
\hline Diffraction source & PAL 7A SB I \\
Wavelength $(\AA)$ & 1.00 \\
Temperature $(\mathrm{K})$ & 100 \\
Detector & ADSC Quantum 270 \\
Crystal-to-detector distance $(\mathrm{mm})$ & 230 \\
Rotation range per image $\left({ }^{\circ}\right)$ & 1 \\
Total rotation range $\left(^{\circ}\right)$ & 90 \\
Exposure time per image (s) & 1 \\
Space group & $I 4_{1} 22$ \\
$a, b, c(\AA)$ & $121.29,121.29,156.06$ \\
$\alpha, \beta, \gamma\left({ }^{\circ}\right)$ & $90,90,90$ \\
Mosaicity $\left({ }^{\circ}\right)$ & 0.126 \\
Resolution range $(\AA)$ & $47.88-2.00(2.10-2.00)$ \\
Total No. of reflections & $284143(41653)$ \\
No. of unique reflections & $39522(5706)$ \\
Completeness $(\%)$ & $99.6(99.7)$ \\
Multiplicity & $7.2(7.3)$ \\
$\langle I / \sigma(I)\rangle$ & $21.8(8.1)$ \\
$R_{\text {meas }}$ & $0.061(0.257)$ \\
Overall $B$ factor from Wilson plot $\left(\AA^{2}\right)$ & 22.9
\end{tabular}

HisTrap column (5 ml; GE Healthcare, Little Chalfont, England) that had been equilibrated with buffer $A$. The column was washed with buffer $A$ and the proteins bound to the HisTrap column were eluted with buffer $B(20 \mathrm{~m} M$ Tris, $130 \mathrm{~m} M \mathrm{NaCl}, 0.2 M$ imidazole $\mathrm{pH}$ 7.5). The extracted active proteins, including ecGAPDH, were loaded onto a HiTrap heparin column (5 ml; GE Healthcare) equilibrated with buffer $A$ (Reisz et al., 2016). After washing the column with buffer $A$, buffer $C(20 \mathrm{~m} M$ Tris $\mathrm{pH} 7.5,1 M \mathrm{NaCl})$ was gradually applied as an elution buffer. ecGAPDH was eluted at concentrations of $200-300 \mathrm{mM} \mathrm{NaCl}$ and was dialysed thoroughly against buffer $A$.

\subsection{Crystallization}

Purified ecGAPDH was concentrated to $20 \mathrm{mg} \mathrm{ml}^{-1}$ using Amicon filters (30 kDa cutoff; Sigma-Aldrich, St Louis, Missouri, USA). Concentrated ecGAPDH $(2 \mu \mathrm{l})$ was mixed with reservoir solution $(2 \mu \mathrm{l})$ consisting of $2.8 \mathrm{M}$ ammonium sulfate, $0.1 M$ 2-( $N$-morpholino)ethanesulfonic acid (MES) $\mathrm{pH} 5.5-6.5$ at $4^{\circ} \mathrm{C}$. GAPDH crystals grew within two weeks using the hanging-drop method. X-ray data were collected from GAPDH crystals treated with a cryoprotectant consisting of $15 \%(\mathrm{v} / \mathrm{v})$ trehalose at $100 \mathrm{~K}$. Crystallization information is given in Table 1 .

\subsection{Data collection and processing}

X-ray diffraction data were collected on beamline 7A at the Pohang Accelerator Laboratory (PAL 7A SB I; Pohang, Republic of Korea) using a CCD detector (ADSC Quantum 270) at an X-ray wavelength of $1.0 \AA$. All images were indexed, integrated and scaled using the $X D S$ program package and the $C C P 4$ program SCALA. The crystals belonged to the tetragonal space group $I 4_{1} 22$, with unit-cell parameters $a=121.29, b=121.29, c=156.06 \AA, \alpha=90, \beta=90$, $\gamma=90^{\circ}$. Data-collection and processing statistics are given in Table 2. 
Table 3

Structure solution and refinement.

\begin{tabular}{ll}
\hline Resolution range $(\AA)$ & $47.7-2.00$ \\
Completeness (\%) & 99.5 \\
No. of reflections & 37461 \\
Final $R_{\text {cryst }}(\%)$ & 18.4 \\
Final $R_{\text {free }}(\%)$ & 20.5 \\
No. of atoms/residues & \\
$\quad$ Protein & $2451 / 330$ \\
Others & $199 / 173$ \\
Sulfate & $5 / 1$ \\
Trehalose & $23 / 1$ \\
Water & $171 / 171$ \\
Total & $2650 / 503$ \\
R.m.s. deviations & \\
Bond lengths $(\AA)$ & 0.010 \\
Bond angles ( $\left.{ }^{\circ}\right)$ & 1.284 \\
Ramachandran plot & \\
$\quad$ Most favoured (\%) & 97 \\
Allowed (\%) & 2.7 \\
Disallowed $(\%)$ & 0.3 \\
\hline
\end{tabular}

\section{Structure determination and refinement}

Initial phases for GAPDH were obtained by a conventional molecular-replacement protocol (rotation, translation and rigid-body fitting) using the structure of E. coli GAPDH (PDB entry 1s7c; Berkeley Structural Genomics Center, unpublished work) as an initial search model. The model was fitted more appropriately by simulated annealing in CNS. Manual rebuilding of the GAPDH model and electron-density interpretation were performed after each refinement cycle using Coot (Emsley et al., 2010). Restrained, individual $B$ factors were refined and the crystal structure was finalized using the $C C P 4$ program REFMAC5 and other programs in the CCP4 suite (Winn et al., 2011). GAPDH was modelled with TLS refinement using anisotropic temperature factors for all atoms. The final model had $R_{\text {free }}$ and $R_{\text {cryst }}$ factors of $20.5 \%$ and $18.4 \%$, respectively (Table 3 ). Structure validation was performed with SFCHECK (Vaguine et al., 1999), PROCHECK (Laskowski et al., 1996) and ADIT (Bhat et al., 2001). DynDom, a program that determines domain movement and relative inter-domain rotation angles in proteins for which two conformations are available, was used to study domain rotation (Poornam et al., 2009; Hayward et al., 1997). Coordinates have been deposited in the Protein Data Bank with accession code $5 \mathrm{za} 0$.

\section{Results and discussion}

GAPDH was purified from E. coli without any genetic manipulation. Despite the use of a nonrecombinant gene, GAPDH could surprisingly be purified using a HisTrap column. Approximately $2 \mathrm{mg}$ of purified ecGAPDH was obtained from a 11 culture. For crystallization purposes, ecGAPDH was concentrated to approximately $20 \mathrm{mg} \mathrm{ml}^{-1}$ using an Amicon concentrator (30 kDa cutoff; SigmaAldrich). Although ecGAPDH was stable at room temperature, crystals suitable for X-ray analysis were grown by the hanging-drop method at $4{ }^{\circ} \mathrm{C}$ (Fig. 1). Tetragonal bipyramidal crystals appeared within two weeks and grew for a further week. For data collection, the crystals were cryoprotected with $15 \%$ trehalose. In the X-ray studies, a single crystal of ecGAPDH was mounted in a cryoloop. The crystals showed significant overloads. Thus, even when the resolution was reduced, X-ray diffraction was measured at $80 \%$ attenuation. Nevertheless, these crystals diffracted to $2.0 \AA$ resolution on PAL 7A SB I (Supplementary Fig. S1).

The structure was solved by molecular replacement using the E. coli GAPDH structure (PDB entry 1s7c) as a starting model. Molecular replacement was performed using Phaser from the $C C P 4$ suite. The final $e c$ GAPDH model had $R_{\text {free }}$ and $R_{\text {cryst }}$ factors of $20.5 \%$ and $18.4 \%$, respectively. The solved structure had a monomer in the asymmetric unit. The monomer contained 330 residues, one sulfate ion, one trehalose molecule and 171 water molecules. The number of water molecules in the current GAPDH structure is restricted despite the high resolution and low mosaicity. To study the conformational changes induced by the binding of trehalose to ecGAPDH, a detailed structural comparison of trehalose-free and trehalose-bound GAPDH was performed.

A list of commonly used sugar cryoprotectants is shown in Supplementary Table S1. Among them, disaccharides such as sucrose, maltose and trehalose have been used to cryoprotect GAPDH crystals. Glycerol, a commonly used cryoprotectant, has also been used in a comparison with sugar-protected GAPDH. Mixtures of 30\% glycerol and 15\% sugars were used for cryoprotection. None of the resulting crystals exhibited any signs of cracking or melting during treatment with the cryoprotectants. The crystals obtained using glycerol as a cryoprotectant showed a resolution of $2.1 \AA$. The crystals obtained using sucrose and maltose showed resolutions of 2.4 and $2.2 \AA$, respectively. The crystals obtained using trehalose showed a resolution of $2.0 \AA$. Although sucrose and maltose have similar structures to that of trehalose, these sugars did not bind to ecGAPDH and glycerol was not found, even though glycerol has previously been reported to be bound in

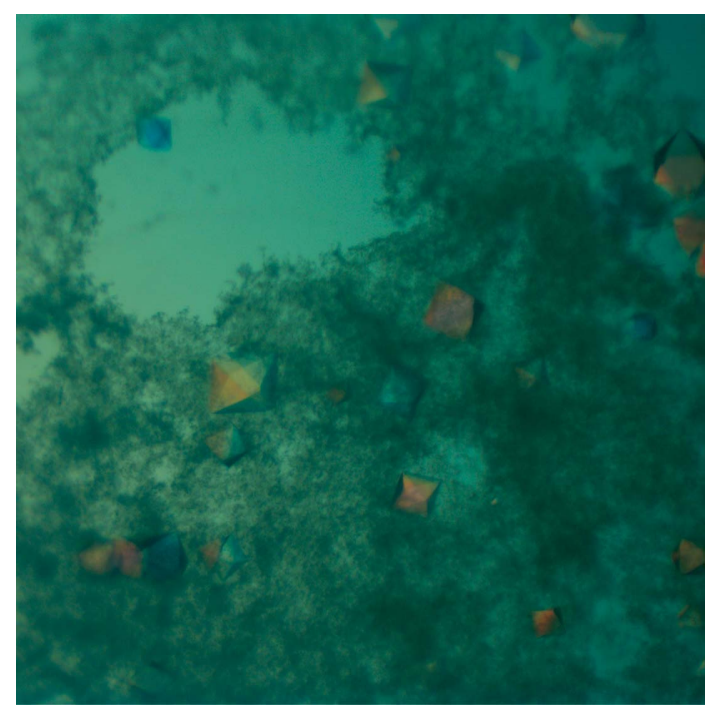

Figure 1

Crystals of $e c \mathrm{GAPDH}$. 
GAPDH structures (Mukherjee et al., 2010; Cook et al., 2009; Robien et al., 2006; Chaikuad et al., 2011; Querol-García et al., 2017; Moreau et al., 2017). Only the GAPDH structure with trehalose was significantly different from published structures.

An electron-density map clearly indicated ordered trehalose molecules bound to the protein. One trehalose molecule bound to ecGAPDH in the S-loop region (Fig. 2). As described above, the S-loop region of GAPDH without $\mathrm{NAD}^{+}$ bound as a cofactor is very flexible and does not exhibit definite electron density (Querol-García et al., 2017). Despite the absence of $\mathrm{NAD}^{+}$, trehalose stabilized the S-loop of the enzyme. As a result, the electron-density map of the S-loop region of the protein was clear. The rotation angle and the relative rotation angle between the $\mathrm{NAD}^{+}$-binding domain and the catalytic domain were measured. The GAPDH molecule shows a rotation of approximately $2.4-3.1^{\circ}$ when superpositioned on the $\mathrm{NAD}^{+}$-free $e c \mathrm{GAPDH}$ (PDB entry $1 \mathrm{dc5}$ ) and/or the $\mathrm{NAD}^{+}$-bound $e c \mathrm{GAPDH}$ (PDB entry 1dc6) structures (Yun et al., 2000; Fig. 3). The inhibition of yeast
GAPDH is reportedly proportional to the concentration of trehalose (Araiza-Olivera et al., 2010). The GAPDHs from yeast and E. coli have similar structures. Although $e c \mathrm{GAPDH}$ has not been reported to be inhibited by trehalose, trehalose induced a conformational change in $e c \mathrm{GAPDH}$ in the current structure. The rotation of GAPDH also induced a conformational change in its active site. This suggests that the binding of trehalose to GAPDH induced a conformational change in its active site to prevent the binding of $\mathrm{NAD}^{+}$, although the $\mathrm{NAD}^{+}$- and trehalose-binding sites differ from one another.

To examine the time dependence of the cryoprotectant effect on GAPDH crystals, the cryoprotectant soaking time was varied from 1 to $10 \mathrm{~min}$ and the data for all crystals from each time were evaluated systematically. The GAPDH crystals neither cracked nor melted as a result of the penetration of cryoprotectants into the solvent channels. These results suggested that the conformational changes in GAPDH owing to the binding of trehalose were independent of soaking time for up to $10 \mathrm{~min}$.

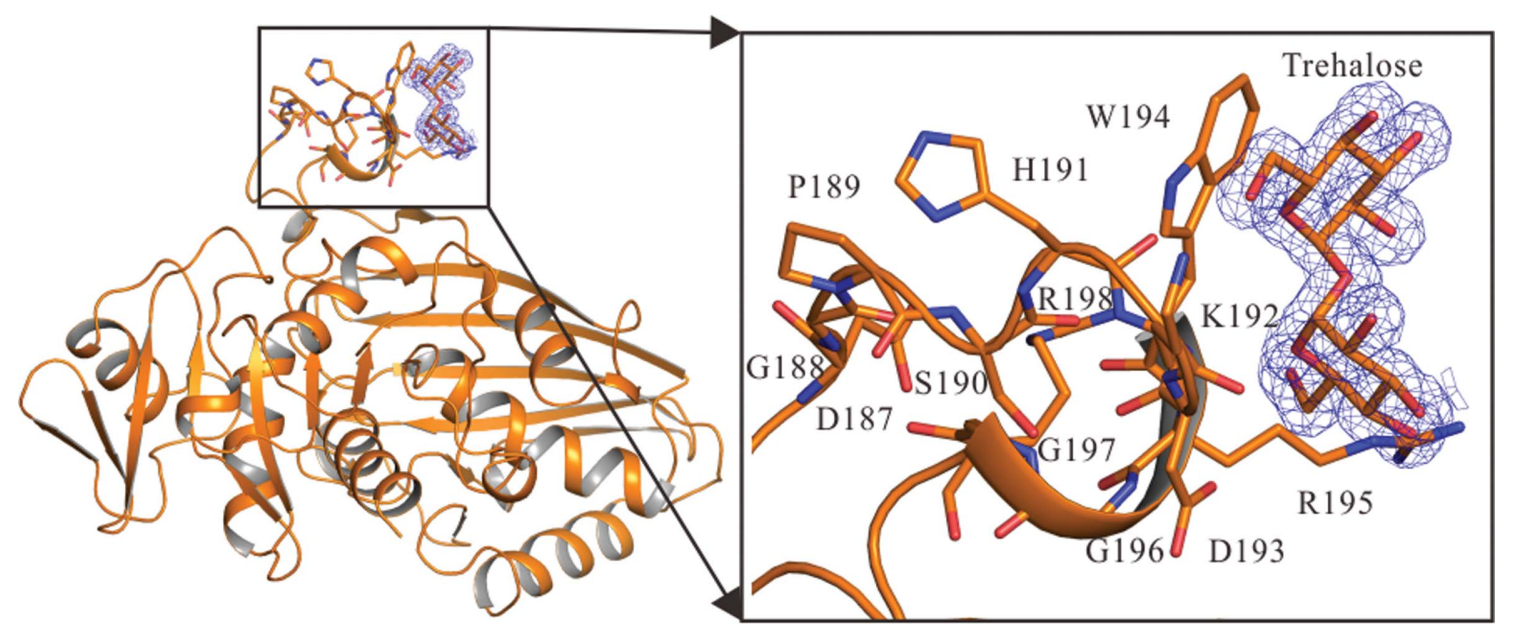

(a)

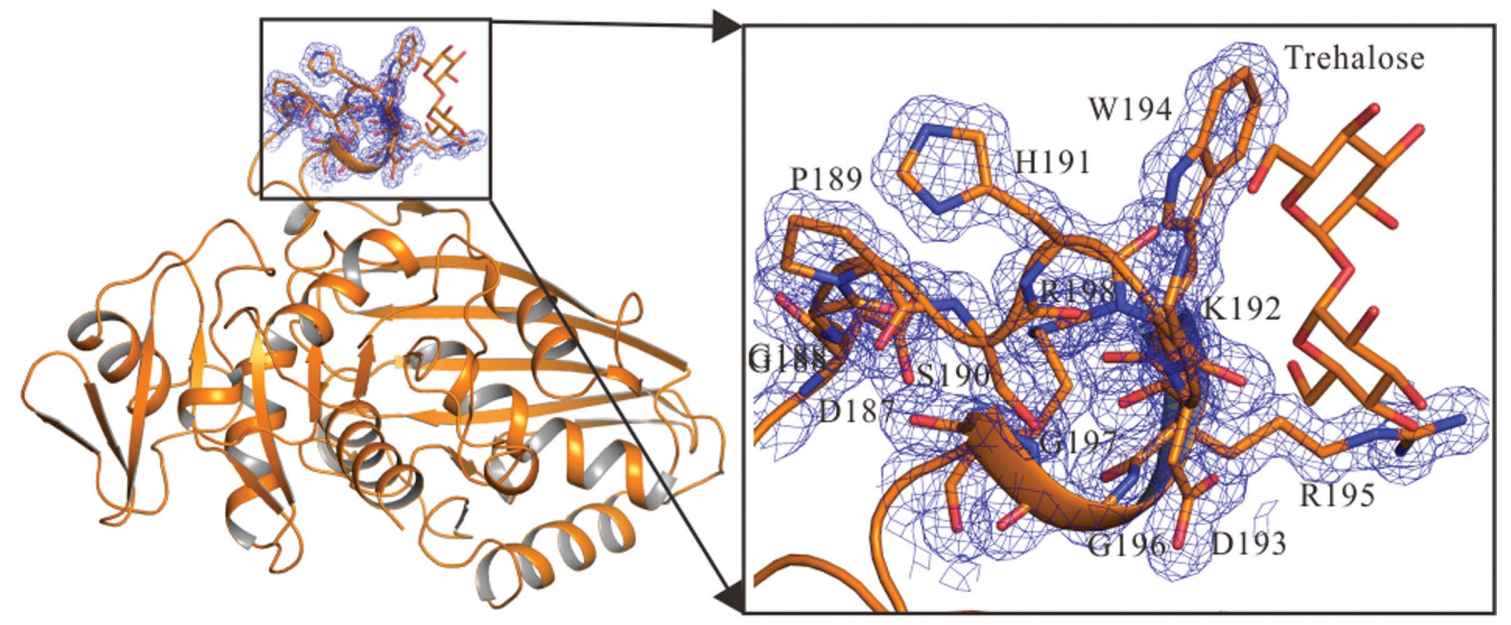

(b)

Figure 2

Electron-density map of trehalose and the ecGAPDH S-loop. ecGAPDH is shown as a cartoon model with trehalose and S-loop residues shown as orange stick models. (a) $2 F_{\mathrm{o}}-F_{\mathrm{c}}$ electron-density map of trehalose contoured at $3.0 \sigma$ (blue mesh). (b) $2 F_{\mathrm{o}}-F_{\mathrm{c}}$ electron-density map of the S-loop residues contoured at $3.0 \sigma$ (blue mesh). 


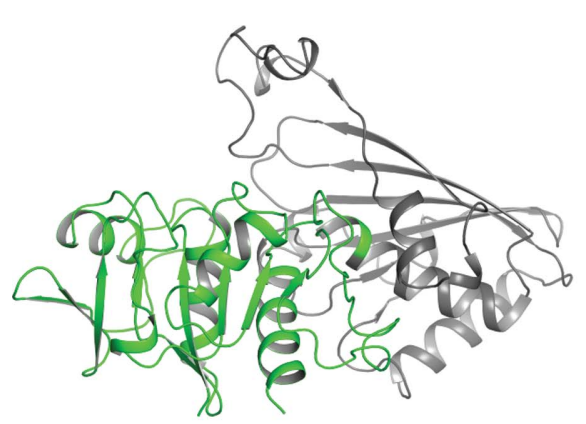

(a)

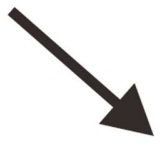

S-loop

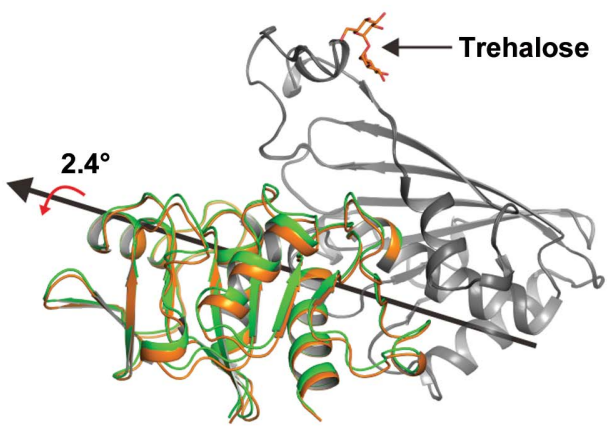

(d)

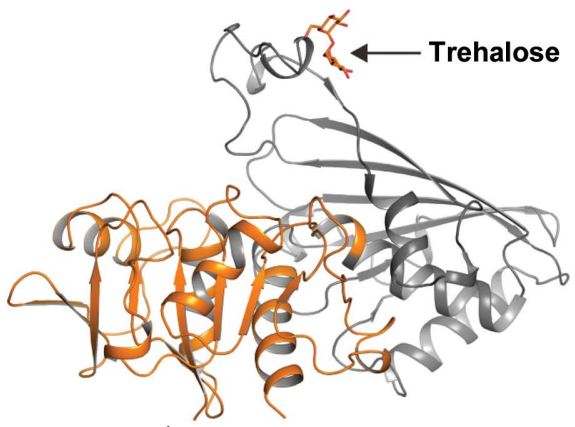

(b)

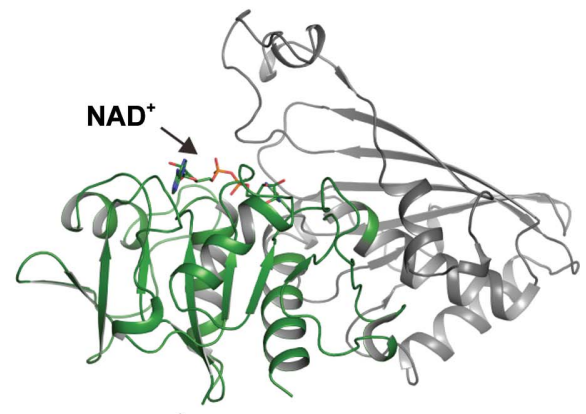

(c)

Figure 3

Rotation of domains in trehalose-bound $e c \mathrm{GAPDH}$ compared with $\mathrm{NAD}^{+}$-free $e c \mathrm{GAPDH}$ and $\mathrm{NAD}^{+}$-bound $e c \mathrm{GAPDH}$. The fixed domain is

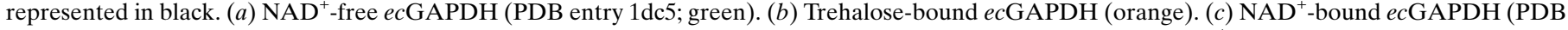
entry 1dc6; forest). (d) The calculated relative rotation angle of trehalose-bound $e c \mathrm{GAPDH}$ compared with NAD ${ }^{+}$-free $e c \mathrm{GAPDH}$ is $2.4^{\circ}$. (e) The calculated relative rotation angle of trehalose-bound $e c \mathrm{GAPDH}$ compared with $\mathrm{NAD}^{+}$-bound $e c \mathrm{GAPDH}$ is $3.1^{\circ}$.

\section{Acknowledgements}

I am grateful to Pohang Accelerator Laboratory (PAL) where the data were collected on beamline 7A. I thank Chair Professor Dr Hui-Woog Choe at Chonbuk National University and at the Department of Brain and Cognitive Sciences of DGIST, Republic of Korea for fruitful discussions and for continuous support of this work. I also thank Professor Dr Joerg Labahn at the Centre for Structural Systems Biology (CSSB), Deutsches Elektronen-Synchrotron (DESY), Hamburg, Germany for discussions during the early stage of this work.

\section{Funding information}

This work was supported by the Basic Science Research Program through the National Research Foundation of Korea (NRF), the Ministry of Science, ICT and Future Planning (2015R1C1A1A02037509), research funds for newly appointed professors of Chonbuk National University in 2014, and a grant from the Jeon-Dae Memorial Fund in 2014.

\section{References}

Araiza-Olivera, D., Sampedro, J. G., Mújica, A., Peña, A. \& UribeCarvajal, S. (2010). FEM. Yeast Res. 10, 282-289.
Baker, B. Y., Shi, W., Wang, B. \& Palczewski, K. (2014). Protein Sci. 23, 1629-1639.

Bhat, T. N., Bourne, P., Feng, Z., Gilliland, G., Jain, S., Ravichandran, V., Schneider, B., Schneider, K., Thanki, N., Weissig, H., Westbrook, J. \& Berman, H. M. (2001). Nucleic Acids Res. 29, 214218.

Chaikuad, A., Shafqat, N., Al-Mokhtar, R., Cameron, G., Clarke, A. R., Brady, R. L., Oppermann, U., Frayne, J. \& Yue, W. W. (2011). Biochem. J. 435, 401-409.

Cook, W. J., Senkovich, O. \& Chattopadhyay, D. (2009). BMC Struct. Biol. 9, 51.

Dastoor, Z. \& Dreyer, J. L. (2001). J. Cell Sci. 114, 1643-1653.

Duée, E., Olivier-Deyris, L., Fanchon, E., Corbier, C., Branlant, G. \& Dideberg, O. (1996). J. Mol. Biol. 257, 814-838.

Emsley, P., Lohkamp, B., Scott, W. G. \& Cowtan, K. (2010). Acta Cryst. D66, 486-501.

Ferreira-da-Silva, F., Pereira, P. J., Gales, L., Roessle, M., Svergun, D. I., Moradas-Ferreira, P. \& Damas, A. M. (2006). J. Biol. Chem. 281, 33433-33440.

Frayne, J., Taylor, A., Cameron, G. \& Hadfield, A. T. (2009). J. Biol. Chem. 284, 22703-22712.

Garman, E. (1999). Acta Cryst. D55, 1641-1653.

Garman, E. (2003). Curr. Opin. Struct. Biol. 13, 545-551.

Hayward, S., Kitao, A. \& Berendsen, H. J. (1997). Proteins, 27, 425437.

Holyoak, T., Fenn, T. D., Wilson, M. A., Moulin, A. G., Ringe, D. \& Petsko, G. A. (2003). Acta Cryst. D59, 2356-2358.

Hope, H. (1990). Annu. Rev. Biophys. Biophys. Chem. 19, 107-126.

Kitatani, T., Nakamura, Y., Wada, K., Kinoshita, T., Tamoi, M., Shigeoka, S. \& Tada, T. (2006). Acta Cryst. F62, 727-730. 
Kosova, A. A., Khodyreva, S. N. \& Lavrik, O. I. (2017). Biochemistry, 82, 643-654.

Laskowski, R. A., Rullmannn, J. A., MacArthur, M. W., Kaptein, R. \& Thornton, J. M. (1996). J. Biomol. NMR, 8, 477-486.

Low, B. W., Chen, C. C. H., Berger, J. E., Singman, L. \& Pletcher, J. F. (1966). Proc. Natl Acad. Sci. USA, 56, 1746-1750.

Moreau, C., Terrasse, R., Thielens, N. M., Vernet, T., Gaboriaud, C. \& Di Guilmi, A. M. (2017). J. Biol. Chem. 292, 2217-2225.

Mukherjee, S., Dutta, D., Saha, B. \& Das, A. K. (2010). J. Mol. Biol. 401, 949-968.

Nicholls, C., Li, H. \& Liu, J.-P. (2012). Clin. Exp. Pharmacol. Physiol. 39, 674-679.

Pflugrath, J. W. (2015). Acta Cryst. F71, 622-642.

Poornam, G. P., Matsumoto, A., Ishida, H. \& Hayward, S. (2009). Proteins, 76, 201-212.

Querol-García, J., Fernández, F. J., Marin, A. V., Gómez, S., Fullà, D., Melchor-Tafur, C., Franco-Hidalgo, V., Albertí, S., Juanhuix, J., Rodríguez de Córdoba, S., Regueiro, J. R. \& Vega, M. C. (2017). Front. Microbiol. 8, 541.

Reisz, J. A., Wither, M. J., Dzieciatkowska, M., Nemkov, T., Issaian, A., Yoshida, T., Dunham, A. J., Hill, R. C., Hansen, K. C. \&
D'Alessandro, A. (2016). Blood, 128, e32-e42.

Robien, M. A. et al. (2006). Proteins, 62, 570-577.

Rodgers, D. W. (1994). Structure, 2, 1135-1140.

Rubinson, K. A., Ladner, J. E., Tordova, M. \& Gilliland, G. L. (2000). Acta Cryst. D56, 996-1001.

Savreux-Lenglet, G., Depauw, S. \& David-Cordonnier, M. H. (2015). Int. J. Mol. Sci. 16, 26555-26581.

Sirover, M. A. (2012). J. Cell. Biochem. 113, 2193-2200.

Vaguine, A. A., Richelle, J. \& Wodak, S. J. (1999). Acta Cryst. D55, 191-205.

Watenpaugh, K. D. (1991). Curr. Opin. Struct. Biol. 1, 10121015.

Winn, M. D. et al. (2011). Acta Cryst. D67, 235-242.

Yun, M., Park, C.-G., Kim, J.-Y. \& Park, H.-W. (2000). Biochemistry, 39, 10702-10710.

Zaid, H., Talior-Volodarsky, I., Antonescu, C., Liu, Z. \& Klip, A. (2009). Biochem. J. 419, 475-484.

Zheng, L., Roeder, R. G. \& Luo, Y. (2003). Cell, 114, 255-266.

Zhou, Y., Yi, X., Stoffer, J. B., Bonafe, N., Gilmore-Hebert, M., McAlpine, J. \& Chambers, S. K. (2008). Mol. Cancer Res. 6, 13751384. 